\title{
Open carpal release using local anesthesia without a tourniquet: Does bleeding tendency affect the outcome?
}

\author{
Seongwon Lee ${ }^{1}$, Sangho $\mathrm{Oh}^{2}$, Daegu Son ${ }^{1}$ \\ ${ }^{1}$ Department of Plastic and Reconstructive Surgery, Keimyung University School of Medicine, Daegu; ${ }^{2}$ W-Hospital, Daegu, Korea
}

Background The aim of this study was to analyze the clinical results of minimal single palmar-incision carpal tunnel release without a tourniquet.

Methods We reviewed the medical records of 75 patients (90 cases of carpal tunnel syndrome) who underwent minimal single-palmar incision carpal tunnel release without a tourniquet from June 2010 to January 2018. Ten patients had a bleeding tendency. We compared the preoperative and postoperative Boston Carpal Tunnel Syndrome Questionnaire (BCTQ) scores. We also analyzed outcomes and complications according to the presence of a bleeding tendency.

Results In all cases, there was a complete disappearance or marked improvement in symptoms within 6 months, with no recurrence. The postoperative BCTQ score showed a significant improvement compared to the preoperative score, and no statistically significant difference in BCTQ scores was detected according to the presence of a bleeding tendency.

Conclusions Carpal tunnel release without a tourniquet using a minimal single palmar incision is effective and reliable. This technique prevents unnecessary pain associated with the tourniquet and is especially helpful in patients with a bleeding tendency or those treated with hemodialysis.

Keywords Carpal tunnel syndrome / Median nerve / Nerve compression syndrome / Hand / Tourniquet
Correspondence: Daegu Son Department of Plastic and Reconstructive Surgery, Keimyung University Dongsan Hospital, Keimyung University School of Medicine, 1035 Dalgubeol-daero, Dalseo-gu, Daegu 42601, Korea Tel: +82-53-258-4583

Fax: +82-53-258-4590

E-mail: handson@dsmc.or.kr

Received: September 1, 2020 • Revised: September 21, $2020 \bullet$ Accepted: October 4, 2020

pISSN: 2234-6163・elSSN: 2234-6171・https://doi.org/10.5999/aps.2020.01732・ Arch Plast Surg 2020;47:597-603

\section{INTRODUCTION}

Carpal tunnel syndrome is the most common peripheral nerve compression neuropathy involving the upper limb. In this condition, the median nerve in the carpal tunnel is compressed, resulting in a tingling or dull sensation in the hand. As nerve compression progresses, it leads to dysesthesia of the median nerve in the dominant region and weakening of the thenar muscle.

The treatment of carpal tunnel syndrome includes conserva- tive procedures such as topical injection of steroids, splinting, and surgery. Surgery is recommended when there is no response to conservative treatment or when symptoms are severe. In 1933, carpal tunnel decompression via transverse carpal ligament (TCL) incision was performed by Learmonth [1]. Although conventional invasive decompression is widely used, it is associated with cicatricial and pillar pain at the surgical site due to the large extent of the incision. To address this issue, minimally invasive techniques such as release with minimal inci- 
sion and endoscopy have become more common. Minimally invasive decompression combines the benefits of both conventional and endoscopic methods. This method ensures prompt recovery after surgery with a minor scar on the skin, and it is safe, as the surgeon has a clear view of the TCL as the incision is performed [2].

Although it is common to use a tourniquet for a clean surgical field, compression by a tourniquet induces additional pain [3]. In patients receiving hemodialysis, the use of a tourniquet is limited in the presence of an ipsilateral arteriovenous fistula in the arm [4]. The wide-awake local anesthesia with no tourniquet (WALANT) technique has recently received attention [5]. In the WALANT technique, no preoperative examination for general anesthesia is needed and there is no discomfort due to tourniquet compression; this technique is also cost-effective and patient satisfaction is high $[6,7]$.

The authors of the present study performed minimally invasive release through local anesthesia without using a tourniquet and compared the preoperative and postoperative Boston Carpal Tunnel Syndrome Questionnaire (BCTQ) scores [8]. In addition, we analyzed the outcomes and complications of patients with and without a bleeding tendency.

\section{METHODS}

\section{Patients}

This retrospective study received approval from the Institutional Review Board of Keimyung University Dongsan Hospital (IRB No. 2020-03-028). This study included 73 patients from June 2010 to February 2018. Of these, 17 patients had bilateral carpal tunnel syndrome, resulting in a total of 90 cases of carpal tunnel syndrome that were treated with single palmar-incision carpal tunnel release without using a tourniquet. Patients with liver cirrhosis, those who had chronic renal failure (with or without dialysis), and those treated with anticoagulants were considered to have a bleeding tendency group. Anticoagulant therapy was discontinued 2 weeks before surgery in those patients.

Based on physical examination, the choice to perform surgical treatment was made for patients who had severe atrophy of the thenar muscle, those who did not respond to persistent conservative treatment, and those with severe sensory depression and pain.

Based on the medical records, we analyzed patients' age and sex, the frequency of left- and right-sided symptoms, and the presence of accompanying diseases. The preoperative and postoperative symptoms, functions, and results were analyzed using BCTQ scores. We also analyzed the outcomes and complica- tions of patients with and without a bleeding tendency.

\section{Surgical procedure}

Surgery was performed without a tourniquet, while the patient lay on a bed with arms abducted and palms turned upwards. The surgical site was sterilized and draped before the surgical design. A Kaplan's cardinal line was drawn on the completely open hand of the patient. An approximately 2 - $\mathrm{cm}$ surgical incision line was drawn starting $1 \mathrm{~cm}$ distal to the wrist crease considering the position of the radial side of the ring finger, the eminence between the thenar and hypothenar muscles, and the longitudinal crease (Fig. 1). To reduce the pain despite local anesthesia and anxiety, intravenous midazolam (midazolam 3 mg/3 mL; Bukwang Pharm Co., Seoul, Korea) and ketamine (ketamine hydrochloride $57.68 \mathrm{mg} / \mathrm{mL}$; Huons Co., Seongnam, Korea) injections were administered. One minute after the sedative injection, we administered $2 \mathrm{~mL}$ of lidocaine mixed with 1:100,000 epinephrine into the surgical site followed by rest for up to 7 minutes. An incision was made along the marked

\section{Fig. 1. Preoperative design}

Kaplan's cardinal and longitudinal lines are drawn from the ulnar side of the middle finger. The expected proximal and distal ends of the transverse carpal ligament are drawn. Based on these lines, the incisional line is drawn.

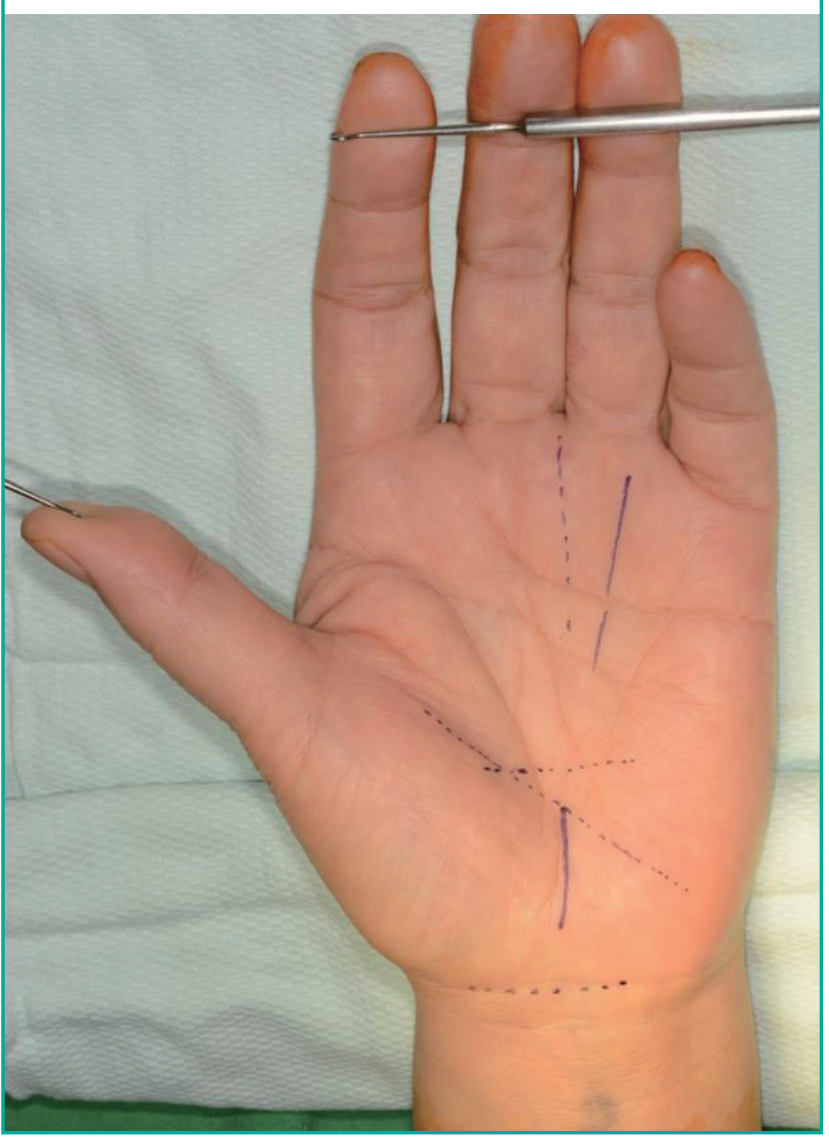


surgical incision line, and the subcutaneous fat and the palmar fascia were spread using blunt Metzenbaum scissors (Fig. 2A). After securing a clear view with a small self-retractor, the TCL was exposed and an incision was made using a no. 15 blade to expose part of the median nerve.

When the TCL was opened by $1-2 \mathrm{~cm}$, the median nerve was pale in color. Subsequently, to release the proximal TCL, the wrist was slightly flexed, and the TCL was separated from the subcutaneous fat for visualization. The proximal portion of the TCL was completely cut using medium-sized Metzenbaum scissors, and dissection was performed using small scissors in the palmar fascia and the soft tissue from the remaining distal part of the TCL, and the ligament was exposed and completely incised (Fig. 2B, completely dissected TCL). The median nerve, which was pale after dissection of the TCL, gradually turned pink as blood flow resumed (Fig. 3, Supplemental Video 1). Finally, the hemorrhage was controlled, and the subcutaneous fat and skin were sutured. After applying a foam dressing, the surgical site was wrapped in a compression bandage with a splint, which was applied to the wrist in a neutral position. The patient's discomfort was minimized by using a short splint (about $10 \mathrm{~cm}$ ). On the day following surgery, the wound was examined in the outpatient office. If no complications were observed, the patient was allowed to change the wound dressing at home. Stitches were removed $7-10$ days postoperatively.

\section{BCTQscores}

Both preoperative and postoperative BCTQ scores were evalu-

\section{Fig. 2. Transverse carpal ligament release}

(A) After dissection, the subcutaneous fat is spread using blunt Metzenbaum scissors and the palmar fascia is seen. (B) The transverse carpal ligament is completely resected.
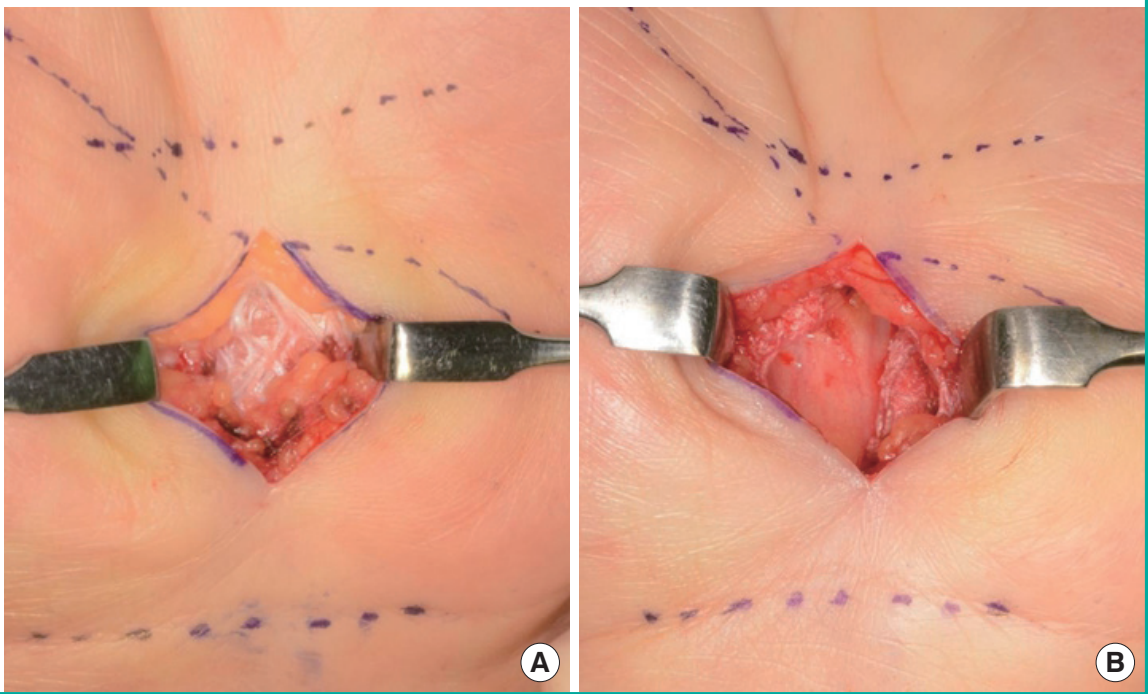

\section{Fig. 3. Median nerve blood flow change}

(A) Immediately after partial release of the transverse carpal ligament (TCL), the median nerve appeared pale. (B) Immediately after complete release of the $\mathrm{TCL}$, the blood flow to the median nerve increased, which could be visualized.
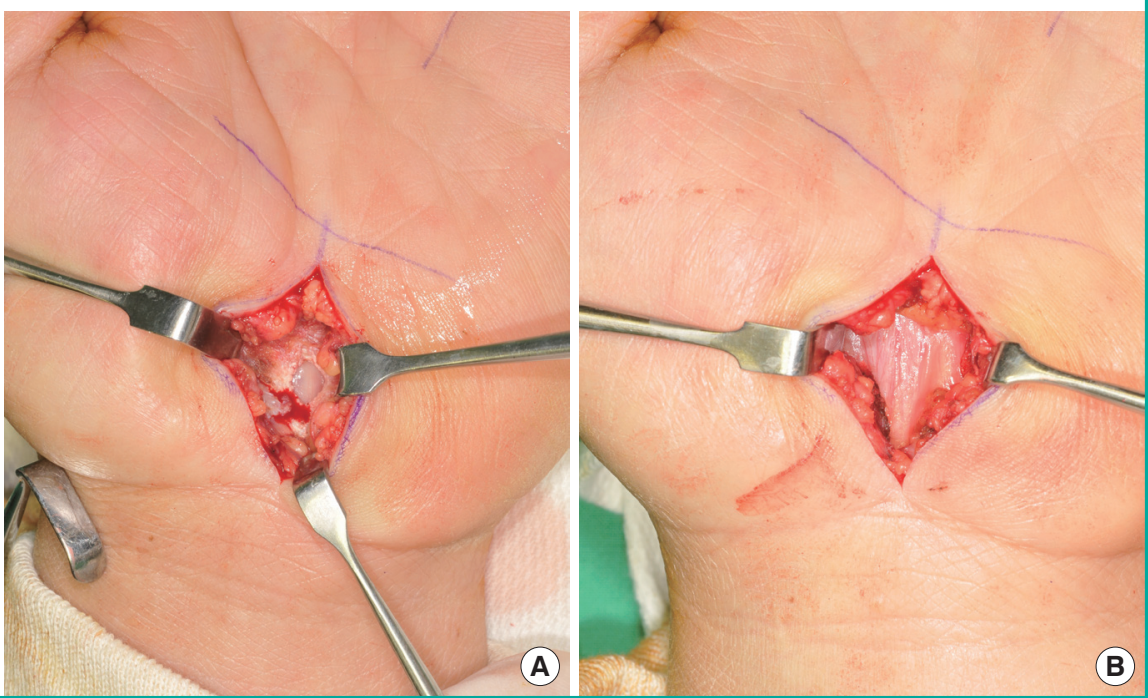
ated. Patients were surveyed preoperatively, 3 weeks after surgery via telephone, and at the final follow-up. The BCTQ consists of a symptom severity scale (SSS) with 11 items and a functional status scale (FSS) with eight items. Each item was assigned 1 point when the patient felt normal or no discomfort, and 5 points when the patient was maximally uncomfortable. After summing the items, the mean score was calculated and the final score was obtained. Forty of the 90 patients were interviewed, and a comparative analysis was conducted based on the SSS, FSS, and BCTQ final scores.

\section{Statistical analysis}

The data were analyzed using the $\mathrm{R}$ stats package, version 3.6.0 (The R Foundation for Statistical Computing Platform, Vienna, Austria). Variables were analyzed using the independent t-test, chi-square test, Fisher exact test, and repeated-measures analysis of variance. P-values of $<0.05$ were considered to indicate statistical significance.

\section{RESULTS}

The study involved 10 men (13 cases) and 65 women ( 77 cases). The mean age at the time of surgery was 59.7 years (range, 36-79 years): three patients (three cases) were in their 30s, five patients (nine cases) in their 40s, 27 patients (31 cases) in their 50 s, 27 patients ( 32 cases) in their 60 s, and 13 patients ( 15 cases) in their 70s. Carpal tunnel syndrome was diagnosed in 34

\begin{tabular}{|c|c|c|c|}
\hline Characteristics & BT group & Non-BT group & P-value \\
\hline No. of cases & 15 & 75 & \\
\hline Age (yr) & $67.5 \pm 10.5$ & $61.2 \pm 10.1$ & $0.002^{\mathrm{a})}$ \\
\hline \multicolumn{4}{|l|}{ Sex } \\
\hline Female & $7(46.7)$ & $69(92.0)$ & $<0.001^{\text {b) }}$ \\
\hline Male & $8(53.3)$ & $6(8.0)$ & \\
\hline \multicolumn{4}{|l|}{ Laterality } \\
\hline Right & $8(53.3)$ & $38(50.7)$ & $0.850^{b)}$ \\
\hline Left & $7(46.7)$ & $37(49.3)$ & \\
\hline Diabetes & $5(33.3)$ & $13(17.3)$ & $0.157^{\text {b) }}$ \\
\hline Hypertension & $13(86.7)$ & $21(28)$ & $<0.001^{\text {b) }}$ \\
\hline NCV & & & $0.023^{c)}$ \\
\hline Mild (grade 2) & 0 & $4(5.3)$ & \\
\hline Moderate (grade 3) & $1(6.7)$ & $23(30.7)$ & \\
\hline Severe (grade 4) & $5(33.3)$ & $9(12.0)$ & \\
\hline Very severe (grade 5) & $2(13.3)$ & $9(12.0)$ & \\
\hline Extremely severe (grade 6) & $2(13.3)$ & $22(29.3)$ & \\
\hline No data & $5(33.3)$ & $8(10.7)$ & \\
\hline Mean follow-up (day) & 33.4 & 103.6 & \\
\hline
\end{tabular}

(37.8\%), 29 (32.2\%), and 27 (30\%) cases involving both hands, the right hand, and the left hand, respectively. Ten patients had a bleeding tendency. Seven of these patients were treated with an anticoagulant; one had liver cirrhosis; and five had chronic renal failure, of whom four received hemodialysis. All cases manifested hand paresthesia and abnormal sensations. Of the 90 cases, 77 were tested preoperatively for nerve conduction velocity, and the results were analyzed using the Bland classification scale [9]. Grade 3 (moderate) and grade 6 (extremely severe) symptoms were the most common, with each occurring in 24 cases (31.2\%), followed by grade 4 (severe) in 14 cases (18.2\%), grade 5 in 11 cases (14.3\%), and grade 2 (mild) in four cases (5.1\%) (Table 1).

Twenty-five cases (28\%) showed symptom improvement within 1 week, 36 cases (40\%) within 1 month, 22 (24\%) within 3 months, and seven (8\%) within 6 months.

Responses to the BCTQ survey was limited to six cases with a bleeding tendency and 34 cases with no bleeding tendency. The demographic characteristics of patients who responded to the BCTQ survey are summarized in Table 2. In the bleeding tendency group, the proportion of men and the severity of nerve conduction velocity were high. In the BCTQ analysis, the patients' SSS score before surgery was $3.89 \pm 0.80$, which gradually decreased to $1.41 \pm 0.95$ at 3 weeks postoperatively and $1.18 \pm$ 0.40 at the final follow-up. The FSS score also decreased gradually from $3.50 \pm 1.61$ before surgery to $1.31 \pm 0.85$ at 3 weeks postoperatively and $1.24 \pm 0.69$ at the final follow-up. The total scores of SSS and FSS improved from $3.70 \pm 1.06$ before surgery to $1.36 \pm 0.86$ at 3 weeks postoperatively and $1.21 \pm 0.52$ at the

Table 2. Demographics of patients participating in the BCTO survey

\begin{tabular}{|c|c|c|c|}
\hline Characteristics & BT group & Non-BT group & P-value \\
\hline No. of cases & 6 & 34 & \\
\hline Age (yr) & $62.7 \pm 5.9$ & $58.3 \pm 8.8$ & $0.247^{a)}$ \\
\hline \multicolumn{4}{|l|}{ Sex } \\
\hline Female & $3(50.0)$ & $33(97.1)$ & \multirow[t]{2}{*}{$0.008^{b)}$} \\
\hline Male & $3(50.0)$ & $1(2.9)$ & \\
\hline \multicolumn{4}{|l|}{ Laterality } \\
\hline Right & $4(66.7)$ & $16(47.1)$ & \multirow[t]{2}{*}{$0.661^{\mathrm{b})}$} \\
\hline Left & $2(33.3)$ & $18(52.9)$ & \\
\hline \multicolumn{3}{|l|}{ NCV } & \multirow[t]{7}{*}{$0.049^{c)}$} \\
\hline Mild (grade 2) & 0 & $3(8,8)$ & \\
\hline Moderate (grade 3) & 0 & $10(29.4)$ & \\
\hline Severe (grade 4) & $3(50.0)$ & $4(11.8)$ & \\
\hline Very severe (grade 5) & $2(33.3)$ & $6(17.7)$ & \\
\hline Extremely severe (grade 6) & 0 & $9(26.5)$ & \\
\hline No data & $1(16.7)$ & $2(5.9)$ & \\
\hline \multicolumn{4}{|c|}{ 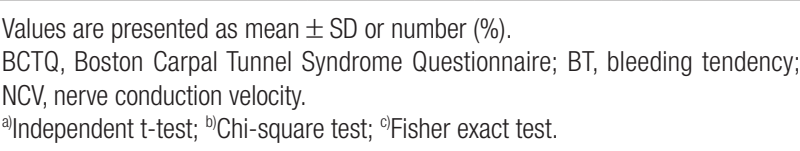 } \\
\hline
\end{tabular}


final follow-up (Table 3). Preoperative and postoperative symptom scores improved in 39 of 40 patients (97.5\%), and functional scores improved in 38 of 40 patients (95\%).

There were three cases of mild complications, including two cases of mild infection and one case of mild widening of the wound. In all cases, conservative treatment was completed and the symptoms did not recur.

We also compared BCTQ scores and complications according to whether patients had a bleeding tendency. The preoperative BCTQ scores did not show a statistically significant difference between the two groups (Table 4). The BCTQ scores improved after surgery in both groups, but no statistically significant between-group difference was detected (Table 5). A minor complication (minimal wound dehiscence) occurred in one of the 15 cases $(6.67 \%)$ in the bleeding tendency group and a mild infection occurred in two of the 75 cases $(2.67 \%)$ in the nonbleeding tendency group.

\section{DISCUSSION}

Studies investigating open or endoscopic release of the carpal tunnel have reported advantages and disadvantages of each

\section{Table 3. Analysis of BCTQ scores}

\begin{tabular}{|lccc|}
\hline Parameter & Preoperative & $\begin{array}{c}\text { 3 } \begin{array}{c}\text { Months after } \\
\text { surgery }^{\text {a) }}\end{array} \\
\text { follow-up }\end{array}$ & $\begin{array}{c}\text { Final } \\
\text { foll }^{\text {a }}\end{array}$ \\
\hline SSS & $3.89 \pm 0.80$ & $1.41 \pm 0.95$ & $1.18 \pm 0.40$ \\
FSS & $3.50 \pm 1.61$ & $1.31 \pm 0.85$ & $1.24 \pm 0.69$ \\
Total & $3.70 \pm 1.06$ & $1.36 \pm 0.86$ & $1.21 \pm 0.52$ \\
\hline
\end{tabular}

Values are presented as mean \pm SD.

BCTQ, Boston Carpal Tunnel Syndrome Questionnaire; SSS, symptom severity scale; FSS, functional status scale.

${ }^{a}$ The results of comparison with preoperative scores (paired t-test) were statistically significant $(P<0.01)$ technique, and it is up to the surgeon to decide the surgical method [10-13]. Carpal tunnel release through a small incision, in which the TCL is incised through a minimal skin incision, alleviates the side effects and challenges of endoscopic decompression. Depending on the number of skin incisions, the technique may entail one or two incisions. The technique using a single incision was introduced by Bromley in 1994 [14]. The TCL was divided under direct vision through a small incision of $1.5-2.0 \mathrm{~cm}$ in the mid-palm. A recent method entails a minimal incision of $2 \mathrm{~cm}$ in the distal wrist crease [15]. The two-incision technique was introduced by Biyani and Downes in 1993 [16] and Wilson in 1994 [17]. This technique allows a surgeon to inspect the median and adjacent nerves via separate incisions in the distal and proximal TCL and to perform an incision while examining the entire ligament. This technique, which involves a broad operative field, has the advantage of preventing incomplete incisions and damage to the peripheral structure, although it leaves behind two scars after surgery.

The authors made a single incision of $2 \mathrm{~cm}$ at $1 \mathrm{~cm}$ distal to the wrist crease, which is the midpoint of the total TCL length. The first incision was made with a no. 15 blade in the middle of the TCL to release $1-1.5 \mathrm{~cm}$ of the ligament under direct vision. Up to this point, the carpal tunnel was not fully released a tourniquet

Table 4. Analysis of the preoperative homogeneity of BCTQ scores

\begin{tabular}{|lccc|}
\hline Parameter & $\begin{array}{c}\text { Bleeding } \\
\text { tendency }\end{array}$ & $\begin{array}{c}\text { No bleeding } \\
\text { tendency }\end{array}$ & P-value \\
\hline SSS & $3.68 \pm 0.66$ & $3.92 \pm 0.82$ & 0.494 \\
FSS & $3.04 \pm 1.27$ & $3.58 \pm 1.67$ & 0.455 \\
Total & $3.36 \pm 0.92$ & $3.76 \pm 1.08$ & 0.408 \\
\hline $\begin{array}{l}\text { Values are presented as mean } \pm \text { SD. } \\
\text { BCTQ, Boston Carpal Tunnel Syndrome } \\
\text { scale; FSS, functional status scale. }\end{array}$ & \\
\hline
\end{tabular}

Table 5. Comparison of BCTQ scores

\begin{tabular}{|c|c|c|c|c|}
\hline Parameter & 3 Months after surgery & Final follow-up & Source & P-value \\
\hline SSS & & & G & 0.644 \\
\hline Bleeding tendency & $1.18 \pm 0.40$ & $1.18 \pm 0.40$ & $\mathrm{~T}$ & 0.414 \\
\hline No bleeding tendency & $1.45 \pm 1.02$ & $1.18 \pm 0.41$ & $G \times T$ & 0.414 \\
\hline FSS & & & G & 0.383 \\
\hline Bleeding tendency & $1.04 \pm 0.10$ & $1.00 \pm 0.00$ & $\mathrm{~T}$ & 0.323 \\
\hline No bleeding tendency & $1.36 \pm 0.92$ & $1.29 \pm 0.74$ & $G \times T$ & 0.801 \\
\hline Total & & & $G$ & 0.475 \\
\hline Bleeding tendency & $1.11 \pm 0.20$ & $1.09 \pm 0.20$ & $\mathrm{~T}$ & 0.371 \\
\hline No bleeding tendency & $1.40 \pm 0.93$ & $1.23 \pm 0.56$ & $\mathrm{G} \times \mathrm{T}$ & 0.485 \\
\hline \multicolumn{5}{|c|}{$\begin{array}{l}\text { Values are presented as mean } \pm \text { SD. } \\
\text { BCTQ, Boston Carpal Tunnel Syndrome Questionnaire; SSS, symptom severity scale; FSS, functional status scale; G, bleeding tendency vs. no bleeding tendency; T, } 3 \text { months } \\
\text { vs. final follow-up; } \mathrm{G} \times \mathrm{T} \text {, interaction for } \mathrm{G} \text { and T. } \\
\text { P-value by repeated-measures analysis of variance. }\end{array}$} \\
\hline
\end{tabular}


was not applied, so the authors were able to observe the median nerve ischemia (Supplemental Video 1) [18]. Local anesthesia was injected into the dermis and subcutaneous fat, and was not administered to the carpal tunnel through the TCL.

Many surgeons use a tourniquet when performing surgical procedures for carpal tunnel syndrome to induce hemostatic effects and to obtain a clear operative field. Recently, Olaiya et al. [19] evaluated the outcomes of wide-awake open carpal tunnel release with and without a tourniquet. According to their systemic review and meta-analysis, the use of a tourniquet induces significantly more pain with no significant clinical benefit when compared with the wide-awake technique in which no tourniquet is used in carpal tunnel decompression.

As the WALANT technique has been used in hand surgery, it has become a safe and effective alternative to carpal tunnel release [20]. The authors administered a sedative immediately before injecting local anesthesia, whereas sedation is not allowed in the WALANT technique. Pain can be reduced in several ways when injecting lidocaine for local anesthesia [21], but the pain following the injection is still uncomfortable for the patient. In a previous study analyzing open carpal release, the WALANT group and the sedation group (monitored anesthesia care, MAC) showed a similar degree of satisfaction, with no statistically significant differences in surgical results [5]. Patients can choose freely between WALANT and MAC. Midazolam and ketamine, used by the authors, are effective and well-established anesthetics commonly used in cosmetic surgery to induce conscious sedation [22]. The authors also found that patients were very comfortable with the surgical outcomes.

Although no tourniquet was used, bleeding was reduced by vasoconstriction induced by epinephrine diluted in lidocaine, and hemostasis was meticulously ensured at bleeding points using a bipolar coagulator to obtain a clear surgical field of view. In particular, in patients with a bleeding tendency, additional bleeding occurs at the skin incision, and comprehensive hemostasis can provide a clear surgical field. If a tourniquet is applied in such a patient, the operation can be performed under a clear field of view, but when the tourniquet is released, the bleeding spreads throughout the surgical field and is difficult to control.

Furthermore, in some patients (e.g., those undergoing hemodialysis), the use of a tourniquet may trigger postoperative shunt thrombosis [23]. Therefore, to avoid this risk, Corradi et al. [4] released an open carpal tunnel without using tourniquets. AlBenna et al. [24] reported that carpal tunnel release without a tourniquet was straightforward as long as suitable hemostatic dissection techniques were used. In this study, all four patients undergoing dialysis in the bleeding tendency group recovered safely, with no complications.
In this study, 15 cases showed a bleeding tendency, but no case of hemorrhage or hematoma after surgery was reported. There was also no statistically significant difference in BCTQ scores according to the presence of a bleeding tendency. We believe that this method is helpful, especially in patients prone to bleeding and those receiving hemodialysis.

In conclusion, carpal tunnel release through a single small incision in the middle of the TCL under local anesthesia without the use of a tourniquet is safe and painless, especially in patients with a bleeding tendency or after hemodialysis. The incision can be made under direct visualization of the TCL through the incision site, which reduces damage to the adjacent structures and minimizes incision site pain, pillar pain, and scar formation.

\section{NOTES}

\section{Conflict of interest}

No potential conflict of interest relevant to this article was reported.

\section{Ethical approval}

The study was approved by the Institutional Review Board of Keimyung University Dongsan Hospital (IRB No. 2020-03028) and performed in accordance with the principles of the Declaration of Helsinki.

\section{Patient consent}

The patients provided written informed consent for the publication and the use of their images.

\section{Author contribution}

Conceptualization: D Son. Formal analysis: S Oh. Project administration: D Son. Writing - original draft: S Lee. Writing - review \& editing: S Oh, D Son.

\section{ORCID}

Seongwon Lee

Sangho Oh

Daegu Son https://orcid.org/0000-0001-9723-3537

https://orcid.org/0000-0002-8739-908X

https://orcid.org/0000-0002-4653-1048

\section{Supplementary material}

Supplemental Video 1. Ischemia reperfusion of the median nerve. The median nerve, which was pale during partial carpal tunnel release, is observed to increase blood flow after complete release. Supplemental data can be found at: https://doi.org/10.5999/ aps.2020.01732.v001. 


\section{REFERENCES}

1. Learmonth JR. The principle of decompression in the treatment of certain diseases of peripheral nerves. Surg Clin North Am 1933;13:905-13.

2. Sur YJ, Song SW, Rhee SK, et al. Carpal tunnel release with minimal single palmar incision. J Korean Soc Surg Hand 2008;13:79-85.

3. Nagpal K, Gossiel M, Kumar H. The impact of tourniquet on patient satisfaction in carpal tunnel decompression. Cent Eur J Med 2007;2:222-6.

4. Corradi M, Paganelli E, Pavesi G. Carpal tunnel syndrome in long-term hemodialyzed patients. J Reconstr Microsurg 1989;5:103-10.

5. Tulipan JE, Kim N, Abboudi J, et al. Open carpal tunnel release outcomes: performed wide awake versus with sedation. J Hand Microsurg 2017;9:74-9.

6. Davison PG, Cobb T, Lalonde DH. The patient's perspective on carpal tunnel surgery related to the type of anesthesia: a prospective cohort study. Hand (N Y) 2013;8:47-53.

7. Lalonde D, Martin A. Tumescent local anesthesia for hand surgery: improved results, cost effectiveness, and wideawake patient satisfaction. Arch Plast Surg 2014;41:312-6.

8. Levine DW, Simmons BP, Koris MJ, et al. A self-administered questionnaire for the assessment of severity of symptoms and functional status in carpal tunnel syndrome. J Bone Joint Surg Am 1993;75:1585-92.

9. Bland JD. A neurophysiological grading scale for carpal tunnel syndrome. Muscle Nerve 2000;23:1280-3.

10. van den Broeke LR, Theuvenet WJ, van Wingerden JJ. Effectiveness of mini-open carpal tunnel release: an outcome study. Arch Plast Surg 2019;46:350-8.

11. Castillo R, Sheth K, Babigian A, et al. Recurrent carpal tunnel syndrome associated with extension of flexor digitorum muscle bellies into the carpal tunnel: a case series. Arch Plast Surg 2018;45:474-8.

12. Varitimidis SE, Herndon JH, Sotereanos DG. Failed endo- scopic carpal tunnel release: operative findings and results of open revision surgery. J Hand Surg Br 1999;24:465-7.

13. Shinya K, Lanzetta M, Conolly WB. Risk and complications in endoscopic carpal tunnel release. J Hand Surg Br 1995; 20:222-7.

14. Bromley GS. Minimal-incision open carpal tunnel decompression. J Hand Surg Am 1994;19:119-20.

15. Yoo HM, Lee KS, Kim JS, et al. Surgical treatment of carpal tunnel syndrome through a minimal incision on the distal wrist crease: an anatomical and clinical study. Arch Plast Surg 2015;42:327-33.

16. Biyani A, Downes EM. An open twin incision technique of carpal tunnel decompression with reduced incidence of scar tenderness. J Hand Surg Br 1993;18:331-4.

17. Wilson KM. Double incision open technique for carpal tunnel release: an alternative to endoscopic release.J Hand Surg Am 1994;19:907-12.

18. Mackinnon SE. Pathophysiology of nerve compression. Hand Clin 2002;18:231-41.

19. Olaiya OR, Alagabi AM, Mbuagbaw L, et al. Carpal tunnel release without a tourniquet: a systematic review and metaanalysis. Plast Reconstr Surg 2020;145:737-44.

20. Sasor SE, Cook JA, Duquette SP, et al. Tourniquet use in wide-awake carpal tunnel release. Hand (N Y) 2020;15:5963.

21. Finsen V. Reduced pain when injecting lidocaine. Tidsskr Nor Laegeforen 2017;137:629-30.

22. Quttainah A, Carlsen L, Voice S, et al. Ketamine-diazepam protocol for intravenous sedation: the cosmetic surgery hospital experience. Can J Plast Surg 2004;12:141-3.

23. Semer NB, Goldberg NH, Cuono CB. Upper extremity entrapment neuropathy and tourniquet use in patients undergoing hemodialysis. J Hand Surg Am 1989;14:897-900.

24. Al-Benna S, Nano PG, El-Enin H. Extended open-carpal tunnel release in renal dialysis patients. Saudi J Kidney Dis Transpl 2012;23:1181-7. 